\title{
The Effects of a Delirium Notification Program on the Clinical Outcomes of the Intensive Care Unit: A Preliminary Pilot Study
}

\author{
Jaesub Park ${ }^{1,2}$, Seung-Taek $\mathrm{Oh}^{1}$, Sunyoung Park ${ }^{2}$, Won-Jung Choi ${ }^{1}$, Cheung Soo Shin ${ }^{3}$, \\ Se Hee $\mathrm{Na}^{3}$, Jae-Jin Kim ${ }^{1,4}$, Jooyoung $\mathrm{Oh}^{5}$, and Jin Young Park ${ }^{1,4}$ \\ 'Department of Psychiatry and Institute of Behavioral Science in Medicine, Yonsei University College of Medicine, Seoul: \\ ${ }^{2}$ Department of Psychiatry, National Health Insurance Service Ilsan Hospital, Goyang; \\ ${ }^{3}$ Department of Anesthesiology, Yonsei University College of Medicine, Seoul; \\ ${ }^{4}$ Department of Psychiatry, Gangnam Severance Hospital, Yonsei University Health System, Seoul; \\ ${ }^{5}$ Department of Biomedical Science and Engineering, Institute of Integrated Technology, Gwangju Institute of Science and Technology, Gwangju, Korea
}

Background: Delirium is common among intensive care unit (ICU) patients, so recent clinical guidelines recommended routine delirium monitoring in the ICU. But, its effect on the patient's clinical outcome is still controversial. In particular, the effect of systems that inform the primary physician of the results of monitoring is largely unknown.

Methods: The delirium notification program using bedside signs and electronic chart notifications was applied to the pre-existing delirium monitoring protocol. Every patient was routinely evaluated for delirium, pain, and anxiety using validated tools. Clinical outcomes, including duration of delirium, ICU stay, and mortality were reviewed and compared for 3 months before and after the program implementation.

Results: There was no significant difference between the two periods of delirium, ICU stay, and mortality. However, anxiety, an important prognostic factor in the ICU survivor's mental health, was significantly reduced and pain tended to decrease.

Conclusions: Increasing the physician's awareness of the patient's mental state by using a notification program could reduce the anxiety of ICU patients even though it may not reduce delirium. The results suggested that the method of delivering the results of monitoring was also an important factor in the success of the delirium monitoring program.

Key Words: anxiety; critical illness; delirium; intensive care units; pain; reminder systems

\section{INTRODUCTION}

Delirium management is still a very complex problem, and therefore the range of pharmacological treatment is limited and the effects of nonpharmacological interventions are controversial [1,2]. Antipsychotic drugs help to manage some specific symptoms of hyperactive delirium, known as intensive care unit (ICU) psychosis [3]. Anxiety and pain can cause delirium, while medications used to relieve these two symptoms can also induce delirium [4,5]. Therefore, it is very difficult to maintain a proper balance. The effects of nonpharmacological treatments have also been controversial.

\footnotetext{
Received on November 27, 2017 Revised on January 14, 2018 Accepted on January 18, 2018

Correspondence to: Jin Young Park, Department of Psychiatry, Gangnam Severance Hospital, Yonsei University Health System, 211 Eonju-ro, Gangnam-gu, Seoul 06273, Korea

Tel: +82-2-2019-3342, Fax: +82-2-2019-4304, E-mail: EMPATHY@yuhs.ac

*No potential conflict of interest relevant to this article was reported.
}

(cc) This is an Open Access article distributed under the terms of the Creative Commons Attribution Non-Commercial License (http://creativecommons.org/ licenses/by-nc/4.0/) which permits unrestricted non-commercial use, distribution, and reproduction in any medium, provided the original work is properly cited. Copyright (c) 2018 The Korean Society of Critical Care Medicine 
Although nonpharmacological interventions such as reorienting communication, oral and nutritional assistance, and early mobilization are known to reduce the incidence and duration of delirium [6], there was no reported effect in a study that provided exercises tailored to individual patient ability, mobilization, and orientation [7]. Furthermore, even if there is improvement in the symptoms of delirium, it is unclear whether this will have a positive impact on prognosis, such as decreased mortality [8].

The principle of treatment for ICU delirium is the correction of the underlying medical condition [9]. Because ICU patients have severe and complex medical problems and often are under various medications, identifying the cause of delirium is often difficult. In clinical practice, it is the primary care physician's thorough evaluation of the patient that usually clarifies the main problem in delirium. Nevertheless, physicians often forget to assess the patient's mental status because they are more focused on the treatment issues in the ICU [10].

The development of delirium has been associated with prolonged hospital stay, increased mortality, and decreased cognitive function in ICU patients [11-13]. In addition, delirium increases the cost of hospitalization, increases the utilization rate of the institute after discharge, and decreases patient social functioning, which increases the caregiver burden and social healthcare costs $[14,15]$. There has been long term clinical interest in the persistent cognitive decline of patients with delirium. Recently, it has been reported that elderly patients who experienced delirium showed symptoms similar to those of dementia $[16,17]$. Accordingly, the quality improvement activities of prevention, early detection, and early management of delirium are gaining widespread use [18,19].

Primary care physicians are often more concerned with factors directly related to mortality in the ICU. These treatment issues require more time and effort. Studies have emphasized the need for better care of delirium in the ICU $[9,20]$. Hence, we have developed a system that informs primary care physicians whether their patients are suffering from delirium. This system has two notification processes. One notification process is conducted by the on-duty nurse who performs a Confusion Assessment Method-ICU (CAM-ICU) on the patient and includes a notification of "CAM-ICU (+)" on the wall just below where the patient's name was printed if the result was positive. A second notification procedure is performed by a psychiatrist who diagnoses the patient as having delirium by using the Diagnostic and Statistical Manual of Mental Disorders (DSM), and indicates the results on the electronic medical record (EMR).

Effective notification of the patient's delirium status to the attending physician in a way that reduces the burden of the ICU staff is important for treatment success [21,22]. Therefore, we established a program to report delirium using this method. Through a more active involvement of a psychiatrist, we hoped to improve the patient's clinical outcome and provide an integrated and continuous view of not only delirium, but also anxiety and pain status. In this study, we hypothesized that (1) increased physician awareness of the delirium condition of the patient through use of a simple notification system improves the clinical outcome of delirium, and (2) the notification system reduces patient distress as measured by the anxiety and pain status.

\section{MATERIALS AND METHODS}

\section{Patient Cohort}

This study was conducted on patients admitted to the general ICU of the tertiary hospital. There were 23 beds for the medical and surgical patients, of which six were isolation rooms. The neonatal ICU, neurological ICU, and cardiac ICU operated separately. The Gangnam Severance Hospital is certified by the Joint Commission International Standards for Hospitals, and the ICU is also operated in accordance with these standards. In particular, the ICU Distress and Delirium Management (IDDM) projects for delirium and distress monitoring of ICU patients have been used since 2012 . 


\section{Delirium Monitoring and Diagnosis Protocols (IDDM Protocol)}

All patients in the ICU were evaluated by a nurse using the Richmond Agitation-Sedation Scale (RASS). The onduty nurses evaluated the patient's delirium using the CAM-ICU $[23,24]$, usually for three times per day, except for comatose patients (RASS score -4 or -5 ). Each day, at approximately 10 AM, based on these data and the direct evaluation of the patients, a psychiatrist confirmed and recorded the patient's delirium condition, dividing the patients into three groups: comatose (RASS score -4 or $-5)$, delirious, and nondelirious/noncomatose. The patients who recovered from delirium, including the nondelirious/noncomatose patients, were evaluated by the psychiatrist, and the patient's distress was assessed and recorded daily, using the anxiety and pain scale.

All patients over the age of 19 years were evaluated from the time of entry to the time of exit. However, the evaluation by the psychiatrist was not performed in patients who had a short ICU stay ( $<24$ hours) or who were not in bed at the scheduled evaluation time. The evaluation results for each patient were recorded in the EMR of each patient. The doctor in charge of each patient read this record, and the doctors of each department directly prescribed or consulted with the psychiatrist.

\section{Assessment of Delirium, Pain, and Anxiety}

The screening of delirium was performed by trained nurses using the CAM-ICU. The CAM-ICU is a widely used screening tool for delirium and its reliability and accuracy have been well documented. All ICU nurses re- ceived CAM-ICU enrollment training at the first job and received at least one retraining each year. In addition, reeducation was conducted by a rounding psychiatrist when necessary. The final diagnosis of delirium was confirmed by a psychiatrist based the DSM-V criteria. To evaluate patient distress, we used the Numeric Rating Scale for Pain (NRS-Pain) [25,26], and the Hamilton Anxiety Rating Scale (HAMA) [27,28]. When using the NRS-Pain scale, patients were asked to score their pain severity between 0 (no pain) and 10 (highest pain) points. This scale has shown acceptable validity in assessing pain among patients in the ICU $[29,30]$. A psychiatrist also assessed anxiety using the HAMA, which is a 14-item scale, and each item score ranged from 0 to 4 . Thus, the total HAMA score varied from 0 to 56 and a score of 0 represented no symptoms, while a higher score represented greater anxiety.

\section{Delirium Notification Program}

Before December 1, 2014, the results of the monitoring program were noticed when the patient's physician read the monitoring sheet on the EMR chart. To improve clinician awareness of their patient's delirium and distress status, two simple methods of notification have been used since December 1, 2014. First, a sign was placed bedside that read "CAM $(+)$ " so that the doctor could observe it when conducting daily ICU rounds. Second, a warning sign was inserted onto the first screen when the physician read the patient's EMR to prescribe, record, or check test results (Figure 1). Physicians were also provided with an electronic chart of the patient's delirium, anxiety, and pain status in a single table, organized by date.
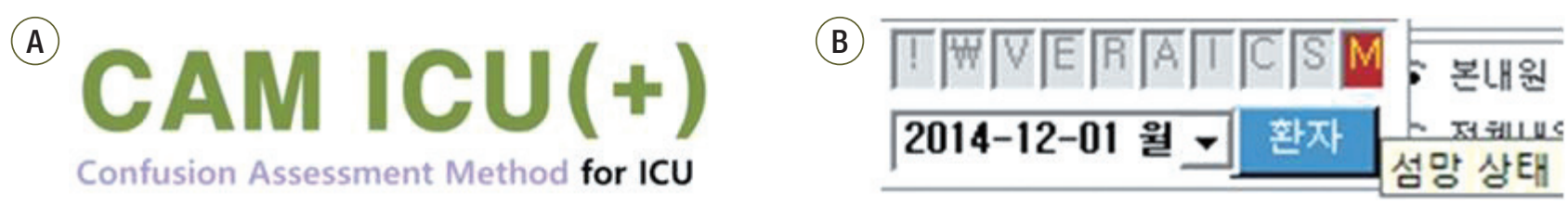

Figure 1. Delirium notification program. (A) Bedside sign: if the patient is confirmed as delirious, the intensive care unit (ICU) nursing staff attached this bedside sign under the patient identification table. When a primary care physician identifies the patient during daily rounding, he or she will see this sign. (B) Warning sign on the electronic medical record (EMR): to see the EMR of a particular patient, a physician must press the blue box. If delirium is present, the delirium warning box will be highlighted in red among a series of alert boxes located above the blue box. When the doctor moves the mouse cursor over the red box, a yellow box with the word "in delirium" will appear. The image is part of the EMR program screen currently in use. CAM: Confusion Assessment Method. 


\section{Study Design}

\section{Patient selection}

To investigate the effects of the simple notification system, we conducted a retrospective comparison study between the before-notification group and after-notification group. For the after-notification group, we collected data from January to March 2015 after a set-up period that was needed to establish the notification system. For the before-notification group, we selected data from January to March 2014. In 2014, we had already evaluated all ICU patients using the same measurement protocols as in 2015, except for the notification system. In Korea, the four seasons are clearly distinguished, therefore, the characteristics of the patient groups could be affected by the seasons. To avoid seasonal bias, we chose the same period, from January to March. Patients were excluded if they stayed in the ICU less than 24 hours (Figure 2).
Because all measurements were part of a daily routine management, no specific informed consent was required. However, during the patient's registration, the patient or caregiver was able to decide whether or not to consent to the use of the medical information for research. We obtained ethical approval for conducting our study from the Institutional Review Board (IRB No. 3-2014-0041).

\section{Data collection}

We reviewed the medical records of patients to obtain demographic information including sex, age, and surgical intervention history. We also obtained the Acute Physiology and Chronic Health Evaluation II (APACHE II) [31] score at ICU admission to estimate the clinical severity of the patients. As the primary outcome measures, the duration of ICU stay, duration of delirium, and mortality in the ICU were reviewed for all patients during each period. Delirium Day was defined as a date when the
(A)

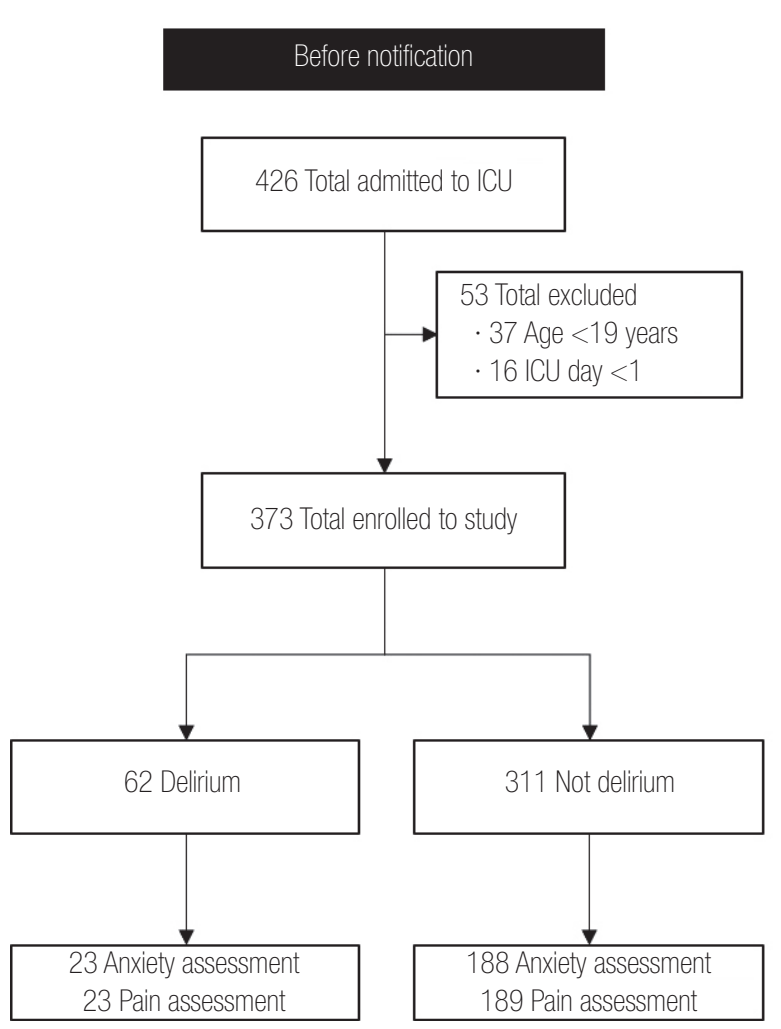

(B)
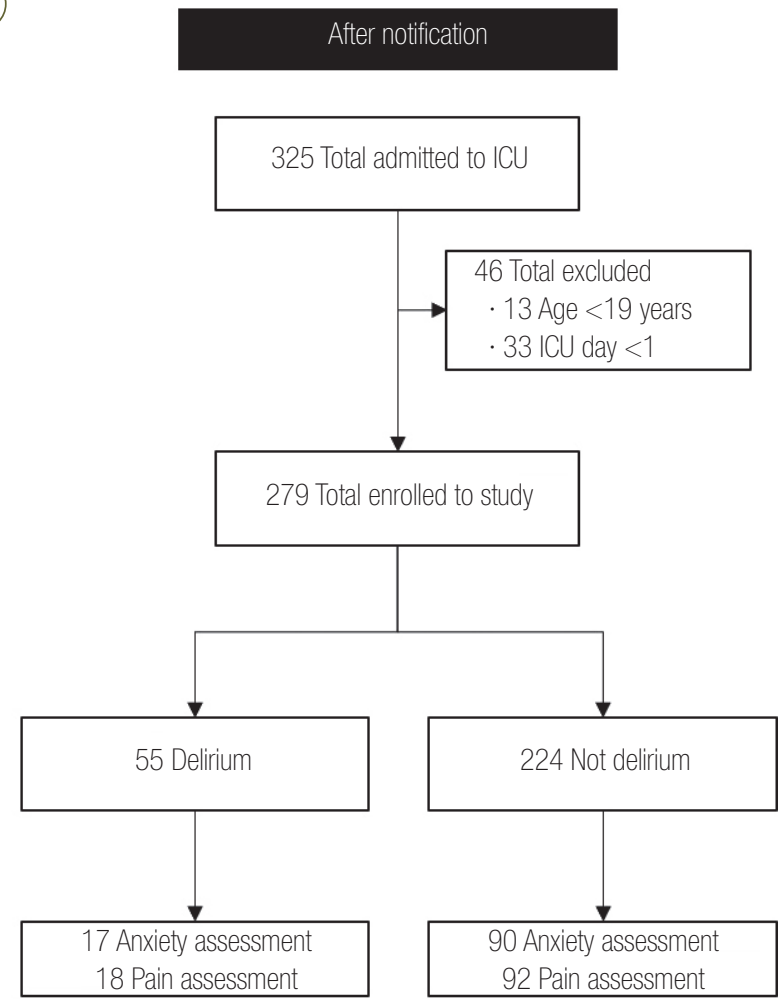

Figure 2. Flowchart of inclusion and subgrouping. (A) Before notification: from January to March 2014. (B) After notification: from January to March 2015. Anxiety was assessed using Hamilton Anxiety Rating Scale and pain was assessed using Numeric Rating Scale for Pain. ICU: intensive care unit. 
CAM-ICU delirium diagnostic criteria were met and a psychiatrist confirmed delirium during the daily rounding. Secondary outcome measures were distress level as measured using the NRS-Pain scale, and anxiety using the HAMA scale. The mean values for these scales were obtained several times during the ICU stay. For additional analyses, the proportion of patients referred to psychiatric consultation among the delirious patients and the proportion of delirium patients who came to outpatient psychiatry after discharge were calculated.

\section{Statistical Methods}

To compare demographic information including sex and operation status, chi-square tests were used. Student t-test was used for comparing age and APACHE score. The delirium rate was compared using Fisher exact test. The univariate analysis of variance was used to compare outcomes, including delirium days, ICU days, and pain and anxiety levels between two periods. In these analyses, age, operation status, and APACHE scores were used as covariants. Mortality rate, the proportion of patients referred to psychiatric consultation, and the proportion of delirium patients who came to the outpatient department were compared using chi-square tests. A P-value $<0.05$ was considered to be statistically significant. Statistical analyses were performed using IBM SPSS version 23.0 (IBM Corp., Armonk, NY, USA).

\section{RESULTS}

We screened 426 ICU patients in 2014 and 325 ICU patients in 2015, and included 373 ICU patients in the before-notification group and 279 ICU patients in the after-notification group for the final analyses. Thirty-seven patients in 2014 and 13 patients in 2015 were excluded because they were younger than 19 years of age. Patients who stayed in the ICU for less than 24 hours were excluded (16 patients in 2014 and 33 patients in 2015).

\section{Clinical Characteristics}

There was no significant difference between the beforenotification group $(\mathrm{n}=373)$ and the after-notification group $(\mathrm{n}=279)$ in age $(64.5 \pm 15.7$ years vs. $63.0 \pm 15.5$ years; $\mathrm{t}=-1.236, \mathrm{P}=0.218)$ and $\operatorname{sex}\left(\chi^{2}=0.00, \mathrm{P}=0.996\right)$. However, there was a significant difference in APACHE scores. The after-notification group showed significantly higher APACHE scores than the before-notification group $(15.0 \pm 9.7$ vs. $12.8 \pm 7.8 ; \mathrm{t}=-3.25 ; \mathrm{P}<0.01)$. The percentage of patients who underwent surgery in the after-notification group was lower than that in the beforenotification group ( $39.8 \%$ vs. $46.9 \% ; \chi^{2}=3.297, \mathrm{P}=0.069$ ) (Table 1).

\section{Primary Outcome}

There was no significant difference between the beforenotification group and the after-notification group in ICU days $\left(5.24 \pm 9.35\right.$ days vs. $5.15 \pm 6.33$ days; $\mathrm{F}_{(1,18.652)}=$ $0.285, \mathrm{P}=0.594)$. The ICU mortality percentage of the after-notification group was slightly higher than that of the before-notification group, but it was not significant $(9.0 \%$ vs. $6.2 \% ; \chi^{2}=1.827 ; \mathrm{P}=0.176$ ). There was no significant difference in delirium percent between the before-notification group and the after-notification group (16.7\% vs. $\left.19.7 \% ; \chi^{2}=1.036 ; \mathrm{P}=0.309\right)$. In the delirium subgroup, there was no significant difference between the beforenotification group and the after-notification group in delirium days (3.03 \pm 4.28 days vs. $3.49 \pm 3.80$ days; $\left.\mathrm{F}_{(1,112.9)}=0.728, \mathrm{P}=0.395\right)$, ICU stay $(11.45 \pm 17.57$ days vs. $9.64 \pm 9.07$ days; $\left.F_{(1,12.164)}=0.559, P=0.456\right)$, and ICU mortality percentage $\left(4.8 \%\right.$ vs. $14.5 \% ; \chi^{2}=3.224, \mathrm{P}=$ 0.073) (Table 1).

\section{Secondary Outcome (Pain and Anxiety)}

The patients in the after-notification group had significantly lower anxiety scores compared with those patients in the before-notification group after adjusting for APACHE score, age, and surgery (11.18 \pm 5.29 vs. 12.30 $\left.\pm 5.59 ; \mathrm{F}_{(1,127.689)}=4.271, \mathrm{P}=0.040\right)$. Patients in the afternotification group showed lower pain levels compared with the patients in the before-notification group, but this 
Table 1. Demographic characteristics and clinical outcomes

\begin{tabular}{|c|c|c|c|c|}
\hline Variable & Before notification $^{a}$ & After notification $^{b}$ & $t / \chi^{2} / F$ & P-value \\
\hline All patients & $(n=373)$ & $(n=279)$ & & \\
\hline \multicolumn{5}{|l|}{ Clinical characteristic } \\
\hline Age (yr) & $64.5 \pm 15.7$ & $63.0 \pm 15.5$ & $t=1.236$ & 0.218 \\
\hline Sex (male:female) & $222: 151$ & $166: 113$ & $\chi^{2}=0.00$ & 0.996 \\
\hline APACHE score & $12.8 \pm 7.8$ & $15.0 \pm 9.7$ & $t=-3.25$ & $<0.01$ \\
\hline Operation $(Y: N)$ & $175: 198$ & $111: 168$ & $\chi^{2}=3.297$ & 0.069 \\
\hline \multicolumn{5}{|l|}{ Clinical outcome } \\
\hline Delirium $\left(\%, n^{9}\right)$ & $16.7(62 / 311)$ & 19.7 (55/224) & $\chi^{2}=1.036$ & 0.309 \\
\hline ICU day & $5.24 \pm 9.35$ & $5.15 \pm 6.33$ & $F=0.285$ & 0.594 \\
\hline ICU mortality $\left(\%, n^{d}\right)$ & $6.2(23 / 350)$ & $9.0(25 / 254)$ & $\chi^{2}=1.827$ & 0.176 \\
\hline Delirious patients & $(n=62)$ & $(n=55)$ & & \\
\hline \multicolumn{5}{|l|}{ Clinical characteristic } \\
\hline Age (yr) & $70.89 \pm 12.48$ & $65.35 \pm 16.80$ & $t=2.004$ & 0.048 \\
\hline Sex (male:female) & $38: 24$ & $36: 19$ & $\chi^{2}=0.217$ & 0.641 \\
\hline APACHE score & $17.44 \pm 7.19$ & $19.02 \pm 9.10$ & $t=-1.035$ & 0.303 \\
\hline Operation (Y:N) & $33: 29$ & $35: 20$ & $\chi^{2}=1.298$ & 0.255 \\
\hline \multicolumn{5}{|l|}{ Clinical outcome } \\
\hline Delirium day & $3.03 \pm 4.28$ & $3.49 \pm 3.80$ & $F=0.728$ & 0.395 \\
\hline ICU day & $11.45 \pm 17.57$ & $9.64 \pm 9.07$ & $F=0.559$ & 0.456 \\
\hline ICU mortality $\left(\%, n^{d}\right)$ & $4.8(3 / 59)$ & $14.5(8 / 47)$ & $\chi^{2}=3.224$ & 0.073 \\
\hline
\end{tabular}

Values are presented as mean \pm standard deviation unless otherwise indicated.

APACHE: Acute Physiology and Chronic Health Evaluation; Y: yes; N: no; ICU: intensive care unit.

a January-March 2014; ' January-March 2015; 'Number of patients with delirium/patients without delirium; 'Number of death/survive.

difference showed only moderate significance after adjusting for APACHE score, age, and surgery $(2.29 \pm 0.80$ vs. $\left.2.67 \pm 2.40 ; F_{(1,13.322)}=3.850, P=0.051\right)$ (Figure 3).

\section{Responses of the Clinicians and Patients}

There was no difference in the percent of delirious patients referred by other physicians to psychiatry between the two groups $\left(6.1 \%\right.$ vs. $\left.6.8 \% ; \chi^{2}=0.14, \mathrm{P}=0.703\right)$. There was also no difference in the percentage of patients visiting the outpatient department after discharge $(0.6 \%$ vs. $\left.1.4 \% ; \chi^{2}=1.10, P=0.294\right)$.

\section{DISCUSSION}

In this study, we applied two simple delirium notification systems to inform the primary care physicians of their patients' mental problems, including delirium, pain, and anxiety symptoms that were confirmed by a psychiatrist. Contrary to our expectations, this study did not confirm our predicted effects of a simple delirium notification system on delirium duration, ICU length of stay, or ICU mortality. However, we found significant reductions in anxiety and a tendency of reduced pain. These results suggested that improvement of the delirium reporting method had a positive effect on reducing patient distress in the ICU. Although survival is the most important problem in the ICU, the psychological state of the patient in the ICU is also an important issue concerning the development of mental illness that may affect the quality of life after discharge $[32,33]$. This study has significance in that it confirmed the possibility of improving mental health of ICU patients by using a simple notification method. 
(A)

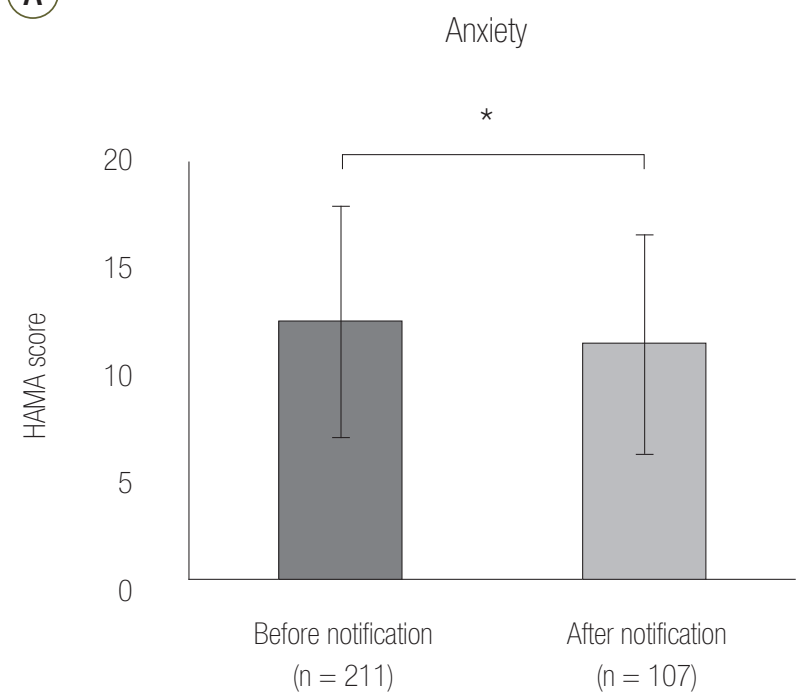

(B)

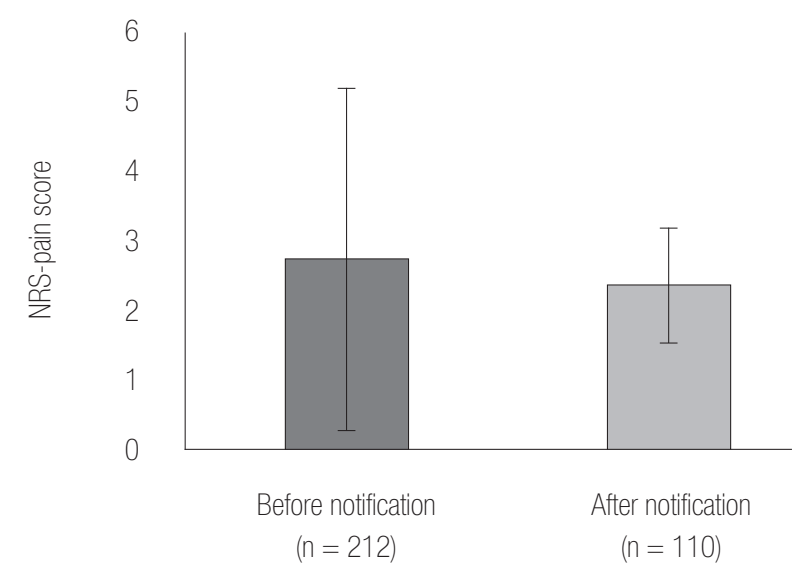

Figure 3. Effects of the delirium notification system on distress. (A) Hamilton Anxiety Rating Scale (HAMA) scores for the before- and after-notification groups $(12.30 \pm 5.59$ vs. $11.18 \pm 5.29 ; F[1,127.689]=4.271 ; P=0.040)$. (B) Numeric Rating Scale for Pain (NRS-Pain) scores for the before- and after-notification groups $(2.67 \pm 2.40$ vs. $2.29 \pm 0.80 ; F[1,13.322]=3.850 ; P=0.051)$. Before-notification group: January-March 2014; After-notification group: January-March 2015. Error bars, ${ }^{*} P<0.05$.

Contrary to our hypothesis, there was no significant difference in duration of delirium, ICU stay, and mortality percentage between the two groups. One possible reason is that despite statistical corrections, an increased delirium severity due to a high APACHE score in 2015 may have offset the statistical effectiveness of the new notification system. It was shown from previous studies that high APACHE scores were predictors of delirium [34,35]. The differences in the APACHE scores also suggested that the characteristics of the inpatient population may differ between the two study periods. Even if the physician became more aware of the patient's delirium, physicians might have had difficulty choosing the appropriate treatment. It may also be difficult to correct the cause of delirium because there are no proven pharmacological [1] or nonpharmacological treatments [2] after delirium has occurred. In addition, the severity of delirium was not measured in this study and only the duration of delirium and mortality percentages during ICU admission were compared. Because there have been treatment studies for delirium that have been shown to reduce symptom severity, even though they did not reduce the duration of the delirium [3], a delirium notification system may have affected the severity of the delirium. Finally, in this study, we measured only one factor, the awareness of the primary physician. However, delirium is a complex disease in which many risk factors work together [36]. Considering previous studies demonstrating that only treatment with multiple factors were effective, it may be difficult to obtain therapeutic effects with a single treatment factor [37].

In this study, there were significant changes in anxiety levels and a trend of pain level reduction after applying the notification system. Before implementation of the notification system, the attending psychiatrist was already routinely checking the patient's anxiety level, thus providing general supportive psychotherapy. In addition, the nurses routinely measured the pain score according to the internal patient management regulations of our ICU. But after implement notification system use, the primary physician was more aware of the patient's anxiety and pain. Therefore, the physicians may have more actively prescribed medication to control the patient's anxiety and pain. Even without additional medications, notice 
of a doctor's concern about their anxiety may have reassured the patient. In our previous study, we showed that the pain level was affected by the psychological state of the patient in the ICU [5]. A physician's reassurance may have stabilized the patient's psychological state and also helped to relieve pain. Recent guidelines state that drugs for anxiety and pain control are recommended [38], and standardized nonpharmacological approaches have also been tried [39]. We did not include routinely prescribed medications or indicated psychological interventions. In the future, it is necessary to determine the most effective methods and their side effects by applying standardized medications and standardized management protocols.

There are some limitations to this study. First, the difference in APACHE scores between the two periods suggested that the clinical features were different, but the analyses did not include the reasons for ICU admission, comorbidities, treatments, types of surgery, and differences in drugs used in the ICU. A very careful interpretation is therefore necessary. However, the higher APACHE II scores after the notification system was used suggested that the positive results of this study were not due to the hospitalization of less severe patients. Second, we did not directly collect the responses of other treatment team members who received notification. It was relatively well known that these delirium monitoring programs improve process outcomes, such as increasing delirium screening adherence and increasing the diagnosis of delirium [20]. In this study, we focused on the impact of clinical outcomes. There was no significant difference in psychiatric counseling between the two periods in this study, which may indicate that therapists ignored it. In the future, studies will need to confirm physicians' responses to the program. Third, although pain and anxiety are related to quality of life as a subjective experience, we did not evaluate the patient's subjective quality of life, and satisfaction with treatment. It is necessary to study whether these improvements in the quality of treatment lead to improvements of patient's satisfaction regarding the reduction of pain and anxiety, and if the system can provide a meaningful treatment for ICU patients.
Treatment of delirium in the ICU is a very complex process involving a large number of people and many factors. ICU practitioners can easily feel helplessness because often small changes in an ICU treatment process may not significantly affect the patient's outcome. However, this study has confirmed that increasing the physician's awareness of patient's mental status by adding a simple notification protocol can lead to a positive change in the patient outcome. When the primary care physician focused on the patient's pain, anxiety, and delirium status, it greatly helped the patient.

\section{Acknowledgments}

We acknowledge Dr. In-Jeong Sohn and Dr. Min-kyeong Kim for their support in the data collection. We also thank the Gangnam Severance Hospital medical records team and the medical information team for extracting data from the electronic medical records. None of the contributors received compensation for their work.

This research was supported, in part, by a grant from the Korea Health Technology R\&D Project through the Korea Health Industry Development Institute, funded by the Ministry of Health and Welfare, Republic of Korea (HI16C0132). This work was also supported by the National Research Foundation of Korea grant funded by the Korea Government (MSIP) (No. 2016R1C1B2010739).

\section{ORCID}

Jaesub Park http://orcid.org/0000-0003-2597-2204

Jooyoung Oh http://orcid.org/0000-0001-6721-399X

Jin Young Park http://orcid.org/0000-0002-5351-9549

\section{REFERENCES}

1. Neufeld KJ, Yue J, Robinson TN, Inouye SK, Needham DM. Antipsychotic medication for prevention and treatment of delirium in hospitalized adults: a systematic review and meta-analysis. J Am Geriatr 
Soc 2016;64:705-14.

2. Hshieh TT, Yue J, Oh E, Puelle M, Dowal S, Travison $\mathrm{T}$, et al. Effectiveness of multicomponent nonpharmacological delirium interventions: a meta-analysis. JAMA Intern Med 2015;175:512-20.

3. Peritogiannis V, Stefanou E, Lixouriotis C, Gkogkos C, Rizos DV. Atypical antipsychotics in the treatment of delirium. Psychiatry Clin Neurosci 2009;63:623-31.

4. Lynch EP, Lazor MA, Gellis JE, Orav J, Goldman L, Marcantonio ER. The impact of postoperative pain on the development of postoperative delirium. Anesth Analg 1998;86:781-5.

5. Oh J, Sohn JH, Shin CS, Na SH, Yoon HJ, Kim JJ, et al. Mutual relationship between anxiety and pain in the intensive care unit and its effect on medications. J Crit Care 2015;30:1043-8.

6. Chen CC, Li HC, Liang JT, Lai IR, Purnomo JD, Yang YT, et al. Effect of a modified hospital elder life program on delirium and length of hospital stay in patients undergoing abdominal surgery: a cluster randomized clinical trial. JAMA Surg 2017;152:827-34.

7. Jeffs KJ, Berlowitz DJ, Grant S, Lawlor V, Graco M, de Morton NA, et al. An enhanced exercise and cognitive programme does not appear to reduce incident delirium in hospitalised patients: a randomised controlled trial. BMJ Open 2013;3:e002569.

8. Al-Qadheeb NS, Balk EM, Fraser GL, Skrobik Y, Riker RR, Kress JP, et al. Randomized ICU trials do not demonstrate an association between interventions that reduce delirium duration and short-term mortality: a systematic review and meta-analysis. Crit Care Med 2014;42:1442-54.

9. Barr J, Fraser GL, Puntillo K, Ely EW, Gelinas C, Dasta JF, et al. Clinical practice guidelines for the management of pain, agitation, and delirium in adult patients in the intensive care unit. Crit Care Med 2013;41:263-306.

10. Palacios-Ceña D, Cachón-Pérez JM, MartínezPiedrola R, Gueita-Rodriguez J, Perez-de-Heredia M, Fernández-de-las-Peñas C. How do doctors and nurses manage delirium in intensive care units? A qualitative study using focus groups. BMJ Open 2016;6:e009678.

11. Ely EW, Shintani A, Truman B, Speroff T, Gordon $\mathrm{SM}$, Harrell FE Jr, et al. Delirium as a predictor of mortality in mechanically ventilated patients in the intensive care unit. JAMA 2004;291:1753-62.

12. Girard TD, Jackson JC, Pandharipande PP, Pun BT, Thompson JL, Shintani AK, et al. Delirium as a predictor of long-term cognitive impairment in survivors of critical illness. Crit Care Med 2010;38:151320.

13. Pisani MA, Kong SY, Kasl SV, Murphy TE, Araujo KL, Van Ness PH. Days of delirium are associated with 1-year mortality in an older intensive care unit population. Am J Respir Crit Care Med 2009;180:1092-7.

14. Leslie DL, Inouye SK. The importance of delirium: economic and societal costs. J Am Geriatr Soc 2011;59 Suppl 2:S241-3.

15. Leslie DL, Marcantonio ER, Zhang Y, Leo-Summers L, Inouye SK. One-year health care costs associated with delirium in the elderly population. Arch Intern Med 2008;168:27-32.

16. Saczynski JS, Marcantonio ER, Quach L, Fong TG, Gross A, Inouye SK, et al. Cognitive trajectories after postoperative delirium. N Engl J Med 2012;367:30-9.

17. Pandharipande PP, Girard TD, Jackson JC, Morandi A, Thompson JL, Pun BT, et al. Long-term cognitive impairment after critical illness. N Engl J Med 2013;369:1306-16.

18. Page VJ, Navarange S, Gama S, McAuley DF. Routine delirium monitoring in a UK critical care unit. Crit Care 2009;13:R16.

19. Luetz A, Weiss B, Boettcher S, Burmeister J, Wernecke KD, Spies C. Routine delirium monitoring is independently associated with a reduction of hospital mortality in critically ill surgical patients: a prospective, observational cohort study. J Crit Care 2016;35:16873.

20. Trogrlić Z, van der Jagt M, Bakker J, Balas MC, Ely EW, van der Voort PH, et al. A systematic review of implementation strategies for assessment, preven- 
tion, and management of ICU delirium and their effect on clinical outcomes. Crit Care 2015;19:157.

21. Andrews L, Silva SG, Kaplan S, Zimbro K. Delirium monitoring and patient outcomes in a general intensive care unit. Am J Crit Care 2015;24:48-56.

22. Jung JH, Lim JH, Kim EJ, An HC, Kang MK, Lee J, et al. The experience of delirium care and clinical feasibility of the CAM-ICU in a Korean ICU. Clin Nurs Res 2013;22:95-111.

23. Ely EW, Margolin R, Francis J, May L, Truman B, Dittus R, et al. Evaluation of delirium in critically ill patients: validation of the confusion assessment method for the intensive care unit (CAM-ICU). Crit Care Med 2001;29:1370-9.

24. Heo EY, Lee BJ, Hahm BJ, Song EH, Lee HA, Yoo $\mathrm{CG}$, et al. Translation and validation of the Korean confusion assessment method for the intensive care unit. BMC Psychiatry 2011;11:94.

25. Hawker GA, Mian S, Kendzerska T, French M. Measures of adult pain: visual analog scale for pain (VAS pain), numeric rating scale for pain (NRS pain), McGill pain questionnaire (MPQ), short-form McGill pain questionnaire (SF-MPQ), chronic pain grade scale (CPGS), short form-36 bodily pain scale (SF-36 BPS), and measure of intermittent and constant osteoarthritis pain (ICOAP). Arthritis Care Res (Hoboken) 2011;63 Suppl 11:S240-52.

26. Farrar JT, Young JP Jr, LaMoreaux L, Werth JL, Poole RM. Clinical importance of changes in chronic pain intensity measured on an 11-point numerical pain rating scale. Pain 2001;94:149-58.

27. Bruss GS, Gruenberg AM, Goldstein RD, Barber JP. Hamilton anxiety rating scale interview guide: joint interview and test-retest methods for interrater reliability. Psychiatry Res 1994;53:191-202.

28. Shear MK, Vander Bilt J, Rucci P, Endicott J, Lydiard B, Otto MW, et al. Reliability and validity of a structured interview guide for the Hamilton Anxiety Rating Scale (SIGH-A). Depress Anxiety 2001;13:166-78.

29. Yu A, Teitelbaum J, Scott J, Gesin G, Russell B, Huynh $\mathrm{T}$, et al. Evaluating pain, sedation, and delirium in the neurologically critically ill-feasibility and reliability of standardized tools: a multi-institutional study. Crit Care Med 2013;41:2002-7.

30. Chanques G, Viel E, Constantin JM, Jung B, de Lattre $\mathrm{S}$, Carr J, et al. The measurement of pain in intensive care unit: comparison of 5 self-report intensity scales. Pain 2010;151:711-21.

31. Knaus WA, Zimmerman JE, Wagner DP, Draper EA, Lawrence DE. APACHE-acute physiology and chronic health evaluation: a physiologically based classification system. Crit Care Med 1981;9:591-7.

32. Girard TD, Shintani AK, Jackson JC, Gordon SM, Pun BT, Henderson MS, et al. Risk factors for posttraumatic stress disorder symptoms following critical illness requiring mechanical ventilation: a prospective cohort study. Crit Care 2007;11:R28.

33. Castillo MI, Cooke ML, Macfarlane B, Aitken LM. Trait anxiety but not state anxiety during critical illness was associated with anxiety and depression over 6 months after ICU. Crit Care Med 2016;44:100-10.

34. Zaal IJ, Devlin JW, Peelen LM, Slooter AJ. A systematic review of risk factors for delirium in the ICU. Crit Care Med 2015;43:40-7.

35. Huai J, Ye X. A meta-analysis of critically ill patients reveals several potential risk factors for delirium. Gen Hosp Psychiatry 2014;36:488-96.

36. Ely EW, Gautam S, Margolin R, Francis J, May L, Speroff $\mathrm{T}$, et al. The impact of delirium in the intensive care unit on hospital length of stay. Intensive Care Med 2001;27:1892-900.

37. Martinez F, Tobar C, Hill N. Preventing delirium: should non-pharmacological, multicomponent interventions be used? A systematic review and metaanalysis of the literature. Age Ageing 2015;44:196204.

38. DAS-Taskforce 2015, Baron R, Binder A, Biniek R, Braune S, Buerkle H, et al. Evidence and consensus based guideline for the management of delirium, analgesia, and sedation in intensive care medicine: revision 2015 (DAS-guideline 2015)-short version. Ger Med Sci 2015;13:19. 
39. Fleischer S, Berg A, Behrens J, Kuss O, Becker R, Horbach A, et al. Does an additional structured information program during the intensive care unit stay re- duce anxiety in ICU patients? A multicenter randomized controlled trial. BMC Anesthesiol 2014;14:48. 